\title{
Psychosocial dimensions of SLE: implications for the health care team
}

\author{
This article was published in the following Dove Press journal: \\ Journal of Multidisciplinary Healthcare \\ 8 April 20II \\ Number of times this article has been viewed
}

\author{
Nancy L Beckerman' \\ Charles Auerbach' \\ Irene Blanco ${ }^{2}$ \\ 'Yeshiva University, Wurzweiler \\ School of Social Work, New York; \\ ${ }^{2}$ Albert Einstein College of Medicine, \\ Bronx, NY, USA
}

Background: The purpose of this exploratory study was threefold, ie, to clarify the unique psychosocial challenges facing those living with systemic lupus erythematosus (SLE), to distinguish which sociodemographic variables impact the lives of SLE patients, and generate knowledge regarding the way patients perceive SLE medication regimens.

Methods: This was a cross-sectional exploratory study in 378 patients diagnosed with SLE and receiving services from the SLE Lupus Foundation in New York City. In addition to sociodemographic variables, the instrument used consisted of two scales, ie, the Systemic Lupus Erythematosus Needs Questionnaire (SLENQ) and the Multidimensional Health Locus of Control Scale, as well as questions regarding subjective perceptions of side effects from SLE medication.

Results: The highest general cause of self-reported depressive and anxious feelings was changes in appearance due to SLE, and limitations in physical abilities due to SLE (primarily from muscle and joint pain). The higher the sense of control over SLE, the less likely respondents were to report feeling depressed and anxious. African-American and Hispanic SLE patients reported a higher level of unmet psychological needs due to SLE than did their other ethnic counterparts. Weight gain and hair loss were the most likely medication side effects and also the most likely causes of SLE-related depression and anxiety.

Conclusion: Those living with SLE are at risk for feelings of depression and anxiety. African-American and Hispanic women are at higher risk for these emotional states. Comprehensive assessment across the disciplines should screen this group of patients for depression and anxiety, and be prepared to refer them to patient education and social work counseling as indicated.

Keywords: lupus, psychosocial impact of illness, multidisciplinary

\section{Introduction}

Systemic lupus erythematosus (SLE) is a chronic autoimmune disease with acute periodic flare-ups of symptoms impacting any organ system and resulting in potentially life-threatening complications. ${ }^{1,2}$ Some of the significant complications of treatment include hirsutism, weight gain, osteoporosis, osteonecrosis, accelerated atherosclerosis, and retinal damage. ${ }^{3,4}$ These side effects and complications can lead to significant functional and emotional challenges. Patients often experience a high degree of psychological symptoms, including anxiety, depression, mood disorders, and decreased health-related quality of life. ${ }^{5-8}$ This article reports on the findings from a preliminary exploratory study on how patients living with SLE perceive their SLErelated challenges. This was a hypothesis-generating study to tease out some of the nuances of the psychosocial challenges for this population.
Correspondence: Nancy L Beckerman Yeshiva University, Wurzweiler School of Social Work, 2495 Amsterdam Avenue, New York, NY I0033, USA Tel + I 2129600839

Fax + I 2I29600822

Email nlbeckerman@gmail.com 
While there are various empirical studies across the health care disciplines on the psychosocial impact of SLE illness, these studies identified general psychosocial experiences without identifying some of the more complex emotional needs of those living with SLE in the US..$^{8-11}$ This study included several instruments, including one that had only been used once previously, ie, the Systemic Lupus Erythematosus Needs Questionnaire (SLENQ) and another known as the Multidimensional Health Locus of Control, that had not ever been used with the SLE population previously. There are many biopsychosocial implications of SLE that have been shown to precipitate depression and anxiety. The disease itself, unexpected exacerbations, medical regimen side effects, and medical care issues are often identified as the sources of depressed feelings. ${ }^{14-18}$ One complex nuance, that has not been addressed fully, is how much does disease activity influence emotional states such as depression and anxiety? And which disease manifestations create the most emotional distress? A patient being treated for disease activity will have medical and nursing needs, and will likely have needs for occupational and physical therapy, as well as social work counseling. ${ }^{8-11}$ The disease disproportionally impacts women (9:1), and women will experience physical changes, such as rashes or a cushingoid appearance, which can trigger feelings of low self-esteem, depression, and anxiety at significantly higher rates than those of healthy women. ${ }^{12-20}$

Danoff-Burg and Friedberg studied the unmet needs of 112 SLE patients. Key findings regarding the impact of SLE included tiredness (94\%), need for assistance about feeling anxious or depressed ( $78 \%$ and $71 \%$, respectively), and nearly half (48\%) desired assistance "related to maintaining relationships with friends". ${ }^{5}$ These findings are consistent with similar international research on the psychosocial impact of SLE. ${ }^{11}$

Moses et al developed and used an SLENQ specifically for 386 SLE patients from a support association in Australia to ascertain their unmet psychosocial needs. ${ }^{12}$ Five of the highest levels of unmet needs were in the psychological domain. They found that "need for help with psychosocial and lifestyle problems outranked the needs for information". ${ }^{12}$ A key implication from this study was that SLE patients should be assessed early on for the likelihood of depressive sequelae.

The current range of multidisciplinary literature indicates that SLE patients have a high vulnerability for self-reported feelings of depression and anxiety. ${ }^{15-19}$ It is unclear which SLE manifestations contribute to the forms of psychosocial distress occurring most often, which sociodemographic cohorts may be at higher risk for such psychosocial distresses, and the nature of the physical and emotional sequelae of SLE medication regimens. We therefore performed a cross-sectional study of 378 SLE patients to identify these psychosocial experiences and which ethnicities may be at risk for which psychosocial stressors.

\section{Materials and methods Participants and procedures}

All 899 individuals in the New York SLE Lupus Foundation contact database were sent the survey instrument. All patients had self-reported having SLE. To protect anonymity, chart reviews were not part of the exploratory process. Each respondent received a six-page survey to investigate their psychosocial experiences of living with SLE. The survey instrument was written at an eighth-grade reading level and was also available in Spanish. The survey was completely anonymous and deidentified. An informed consent letter was sent along with each survey that explained the purpose of the study, its voluntary nature, that participants could discontinue without any penalty, and that the information would be used in the aggregate with no identifying information. To maintain the anonymity of patients' responses, survey completion was used instead of a signature on the informed consent letter. Packets were distributed to home mailing addresses with stamped envelopes, so that completed surveys could be bulkmailed to the researchers at Yeshiva University with complete anonymity. Of the 899 questionnaires originally distributed, 19 were returned unopened due to a change of address. Of 880 received, a total of 378 individuals responded to the survey in English or Spanish (336 in English and 42 in Spanish), with an overall return rate of $42.9 \%$.

\section{Instrument}

The survey instrument comprised four components. Part one included sociodemographic variables including gender, race, and age, as well as time of diagnosis, length of diagnosis, education, employment, and relationship status.

Part two consisted of two scales, ie, the SLENQ, developed and validated by Moses et al, ${ }^{12}$ which uses a 5-point Likert scale $(1=$ no need, $3=$ moderate need, $5=$ high need) for 12 different psychosocial factors. For example, the SLENQ asks: "How much assistance do you need with your change in appearance due to SLE?" and "How much assistance do you need because you have anxiety about SLE". ${ }^{12}$ For the purpose of analysis, three subscales were created from the psychosocial need items, ie, depression, anxiety, and socioeconomic coping. Depression was assessed by the following 
items: feeling depressed due to limitations caused by SLE; feeling depressed because of changes in the body; and feeling depressed because of side effects. Anxiety regarding SLE was assessed by the following items: feeling confused about why this disease happened to you; anxiety about changes in your appearance; feeling angry about having SLE; feeling uncertain about the future; dealing with anxiety about SLE; and anxiety about side effects. Socioeconomic coping consisted of the following items: concerns about gaining employment; satisfactory performance in job; and coping with extra costs. The SLENQ subscales have been validated, with higher scores reflecting higher need. ${ }^{11}$ Reliability of the subscales was high, with coefficent alphas of 0.91 for depression, 0.90 for anxiety, and 0.76 for economic coping. One-way analysis of variance was utilized to test how various factors like age, educational level, employment, and race impact psychosocial need.

In part three, the second scale was used, ie, the Multidimensional Health Locus of Control Scale measuring the respondents' subjective perceptions of how much control they had over their SLE. ${ }^{21}$ Two subscales, ie, "chance" and "internal", were utilized in this research. Chance refers to the mindset that the course of one's illness is out of one's control. Internal refers to the opposite perspective, ie, "If I manage my illness with diet, exercise, compliance with medication regimens, I can control its course". Each is a six-item selfreport questionnaire that uses a 6-point Likert scale, with items ranging from 1 (disagree very much) to 6 (agree very much). Examples of items included in the chance subscale are: "No matter what I do, I am going to get sick", and "Most things that affect my health happen to me by accident". Examples of items included in the internal subscale are: "If I get sick, it is my own behavior which determines how soon I get well again" and "I am in control of my health". It is important to note that the subscales are independent of each other. The internal reliability for these subscales was good, with a coefficent alpha of 0.76 for chance and 0.77 for internal. Each subscale can range between 1 (lowest need) and 6 (highest need).

Part four concluded with open-ended questions about the range of medication regimens, side effects, and psychosocial impact of those medication regimens.

\section{Statistical analysis}

The data were analyzed using SPSS (v 17.0; SPSS, Inc, Chicago, IL) and STATA (v 11.0; Stata Corp, College Station, TX). Statistical tests used in this analysis included the Chi-square, $t$-test, and analysis of variance. Ordinary least squares regression was used to analyze the Likert scale questions on the SLENQ and Multidimensional Health Locus of Control scales. Ordinary least squares regression was used to perform a multivariate analysis in order to evaluate how the different variables affected the outcome measures of depression, anxiety, and socioeconomic coping derived from the SLENQ. The indicators "internal" and "chance" were entered as continuous variables. African-American, Hispanic, and Asian were contrasted with White; education was coded as some college, college, and advanced degree, and contrasted with high school or less education. How the respondents rated their experience with SLE was also coded as chronic symptoms and frequent flares, and contrasted with infrequent flares. Finally, insurance was coded as Medicaid, Medicare, and no insurance, and contrasted with private insurance. "Coef" in Table 2 indicates the slope which shows how much the degree of an outcome variable (depression, anxiety, or socioeconomic coping) changes for every point increase in a covariate (chance, internal). For example, when "chance" increases by 1, a respondent's level of depression increases by 0.17 points. A respondent who had a score of 5 on this scale would have a 0.85 -point increase $(5 \times 0.17)$ in their degree of depression. Postestimation Wald tests were utilized to test the significance of indicator variables, such as race and insurance. With regards to missing data, some respondents did not respond to every question, so some items were tabulated with less than the total number of respondents. List-wise removal of missing data was utilized because missing cases were not missing at random.

Each of the subscales on the SLENQ, ie, depression, anxiety, and socioeconomic coping, ranges from 1 (no need) to 5 (high need). It has been reported that “ $\ldots$ formal statistical tests for normality are especially undesirable as they will have low power in the small samples where the distribution matters and high power only in large samples where the distribution is unimportant". ${ }^{22}$ Given the relatively large sample size of nearly 400 subjects reported here, the means and the medians were compared for overall skew. When the mean and median are equal, it indicates that the distribution is symmetrical. The criterion of symmetry is met for each scale. Parametric tests perform well with large samples (more than 100) even when the data are non-normal. ${ }^{23}$ As a result, the $t$-test and one-way analysis of variance were utilized to compare groups of respondents. The criterion for statistical significance in this study was an alpha level of 0.05 .

Regarding ordinary least squares regression analysis, each of the regression models was tested for normality of residuals, homoscedasticity, multicollinearity, model specification, 
and linearity. The residuals in all three models deviated slightly from normality. Lumley et al point out that “... linear regression does not require any assumption of normal distribution in sufficiently large samples. Previous simulations studies show that 'sufficiently large' is often fewer than $100 \ldots .22$ The Breusch-Pagan test for heteroskedasticity was conducted for the models. The results were nonsignificant, indicating homogeneity of their respective residuals. The variance inflation factor was calculated to test for the presence of collinearity in each model. All models had mean variance inflation factors close to 1 , and no independent variable had a variance inflation factor above 2 , indicating that the independence assumption was met. The models were tested for model specification errors, which can inflate regression coefficents. This type of error occurs when a relevant variable is omitted or an irrelevant one is included. The Ramsey RESET Test was utilized to test for this type of error. The results indicated that the coefficents in each of the models were not influenced by a model specification error. Finally, by utilizing scattergrams between the outcome variables and key independent variables, the models met the linearity assumption.

\section{Results \\ Sample characteristics}

As expected, the vast majority of respondents $(n=357$, $96.5 \%$ ) were women. Age ranged from 20 to over 67 years, with approximately one-third under 35 years $(n=97,26 \%)$, one-third aged $36-45$ years $(n=100,27 \%)$, and one-third aged $\geq 46$ years $(n=123,33 \%)$. The majority of respondents were non-White women, with 40\% $(\mathrm{n}=144)$ identifying themselves as African-American and 38\% $(\mathrm{n}=135)$ as Hispanic. A large majority of the group was either unemployed $(19.4 \%)$ or receiving disability due to SLE (44\%). Most of the respondents $(70.4 \%)$ had been diagnosed with SLE more than 5 years earlier and, in the last 12 months, more than one-third $(37.3 \%, \mathrm{n}=139)$ had been hospitalized because of complications from SLE. The most frequent type of medical coverage for the respondents was Medicaid (44.7\%, $n=168)$, followed by private coverage (29.1\%). The primary source of medical care for the majority of respondents was provided by a private physician $(53.95 \%, \mathrm{n}=191)$ followed by clinics $(37.9 \%, n=134)$. Further demographic and socioeconomic data are listed in Table 1 . The sociodemographic variables of this sample were representative of the national profile of this population regarding age, race, and ethnicity. ${ }^{19}$ The only significant divergence was for education. This sample had a higher level of education than what is reported in most lupus studies..$^{6-11}$
Table I Demographics of a sample with systemic lupus erythematosus $(n=378)$

\begin{tabular}{|c|c|c|}
\hline & $\%$ & $\mathbf{n}$ \\
\hline \multicolumn{3}{|l|}{ Gender } \\
\hline Male & 3.5 & 13 \\
\hline Female & 96.5 & 367 \\
\hline \multicolumn{3}{|l|}{ Race/ethnicity } \\
\hline Hispanic & 37.7 & 135 \\
\hline African-American & 40.2 & 144 \\
\hline Asian & 4.7 & 17 \\
\hline White & 17.3 & 62 \\
\hline \multicolumn{3}{|l|}{ Age (year) } \\
\hline$<21$ & 3.2 & 12 \\
\hline $21-35$ & 26.1 & 97 \\
\hline $36-45$ & 26.9 & 100 \\
\hline $46-60$ & 33.1 & 123 \\
\hline$\geq 61$ & 10.8 & 40 \\
\hline \multicolumn{3}{|l|}{ Education level } \\
\hline High school or less & 27.4 & 102 \\
\hline Some college & 33.9 & 126 \\
\hline College graduate & 29 & 108 \\
\hline Advanced degree & 9.7 & 36 \\
\hline \multicolumn{3}{|l|}{ Employment } \\
\hline Part time & 12.2 & 44 \\
\hline Full time & 24.4 & 88 \\
\hline Unemployed & 19.4 & 70 \\
\hline On disability & 44 & 159 \\
\hline \multicolumn{3}{|l|}{ Insurance } \\
\hline Medicaid & 44.7 & 155 \\
\hline Medicare & 17.9 & 62 \\
\hline Private insurance & 29.1 & 101 \\
\hline None & 8.4 & 29 \\
\hline
\end{tabular}

Two hundred and twenty-eight (60.58\%) respondents indicated that their SLE was marked by a chronic set of symptoms. Another $16.23 \%(\mathrm{n}=61)$ had frequent flares, while $19.13 \%(n=72)$ reported infrequent flares. Joint aches, fatigue, and muscle pain were present for at least two-thirds of the respondents. Respondents reporting chronic symptoms or frequent flares had higher psychosocial needs as determined by their mean scores, ie, depression ( $3.8 \pm 1.1$, $P=0.000)$, anxiety $(3.7 \pm 1.1, P=0.000)$, and socioeconomic coping $(3.3 \pm 1.2, P=0.043)$, as compared with those having infrequent flares. The level of detail was fairly broad. Specific disease manifestations, such as retinal damage and renal damage, did not emerge.

\section{SLE needs questionnaire}

Participants responded concerning their level of psychosocial needs using the SLENQ, as previously detailed. Figure 1 displays the median scores for each factor from lowest to highest need for psychosocial support or assistance. Needing some form of psychosocial assistance for coping with their feelings 


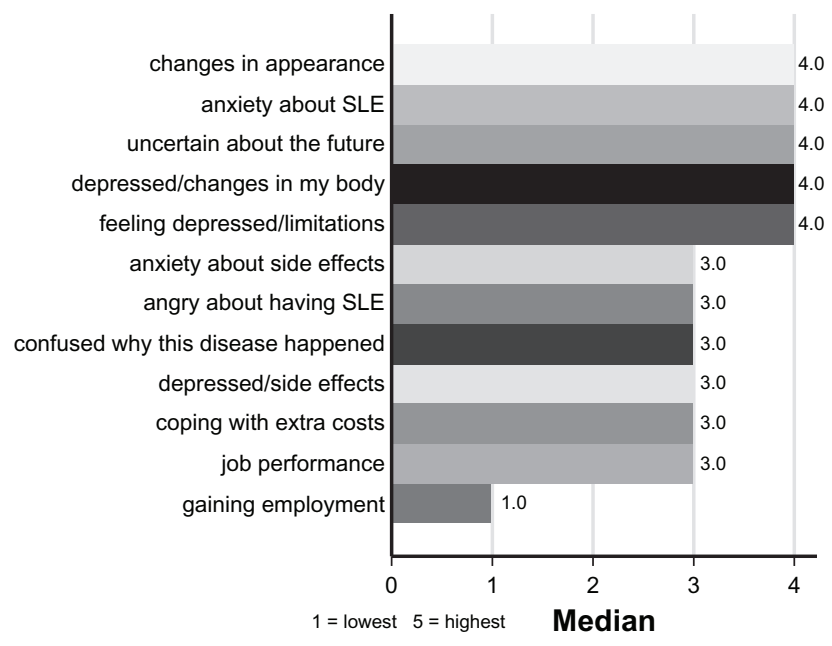

Figure I Psychosocial problems.

of "depression because of changes in body" and "changes in appearance" was rated as their highest concern.

Each of the subscales, ie, depression, anxiety, and socioeconomic coping, ranged from 1 (no need) to 5 (high need). The scales had the following overall means and medians: depression mean $=3.5 \pm 1.3$, median $=3.7$, interquartile range $=2$; anxiety mean $=3.3 \pm 1.2$, median $=3.3$, interquartile range $=2$; and socioeconomic coping mean $=2.9 \pm 1.3$, median $=2.7$, interquartile range $=2.3$. The means and medians indicate that respondents had the most difficulty coping with depression, followed by anxiety and socioeconomic coping. Lumley et al point out that respondents reporting chronic symptoms or frequent flares are more likely to have higher psychosocial needs with their depression, anxiety, and socioeconomic coping, as compared with those having infrequent flares. ${ }^{22}$ Those who reported frequent flares had a mean score of $3.8 \pm 1.1$ for depression $(P=0.000)$, a mean of $3.7 \pm 1.1$ for anxiety $(P=0.000)$, and a mean of $3.3 \pm 1.2(P=0.043)$. Respondents reporting chronic symptoms also reported significantly higher psychosocial needs on depression and anxiety compared with those reporting infrequent symptoms. The means were $3.6 \pm 1.2$ and $3.4 \pm 1.2$, respectively.

Education impacts the level of perceived psychosocial need, mediating levels of self-reported depression and anxiety associated with SLE. For depression, respondents with a high school education or lower rated their psychosocial need as $4.0 \pm 1.1$ compared with those who obtained college or advanced degrees $(3.2 \pm 1.3$ and $3.0 \pm 1.5$, respectively, $P<0.001)$. A similar pattern existed for anxiety, where respondents with high school education or less had an average need of $3.8 \pm 1.2$ compared with $3.0 \pm 1.2$ for respondents who had obtained at least a college degree $(P=0.001)$.

Respondents who were unemployed or receiving disability insurance had higher psychosocial needs on all three subscales. Those who were more fully employed reported less distress with depression, anxiety, and socioeconomic coping. For depression, respondents on disability rated their need as $3.8 \pm 1.2$ compared with $3.1 \pm 1.4$ and $3.1 \pm 1.3$ for those working part time or full time $(P<0.001)$. A similar pattern was true for anxiety, where respondents receiving disability had a mean of $3.6 \pm 1.2$ compared with $3.0 \pm 1.2$ and $3.1 \pm 1.2$ for respondents working full time and part time, respectively $(P=0.001)$.

Hispanic respondents demonstrated the highest need for psychosocial assistance on all three subscales. For feelings of depression, Hispanic respondents rated their need for assistance as $3.8 \pm 1.2$ compared with $3.5 \pm 3.5$ for African-Americans, $2.9 \pm 1.3$ for Asians, and $3.2 \pm 1.4$ for White respondents $(P=0.009)$. On anxiety, Hispanic respondents rated their need for psychosocial assistance as $3.5 \pm 1.2$ compared with $2.8 \pm 1.1$ for Asians, $3.3 \pm 1.2$ for African-Americans and $3.0 \pm 1.2$ for Whites $(P=0.013)$. For socioeconomic coping, White respondents displayed little need for assistance, with a mean of $2.3 \pm 2.3$ compared with $3.1 \pm 1.3$ for Hispanics, $2.9 \pm 1.3$ for African-Americans, and $3.0 \pm 1.0$ for Asians. Respondents on Medicaid rated their psychosocial needs highest on the depression and anxiety subscales. Those who indicated a lack of insurance rated the highest need for assistance with socioeconomic coping. The mean on the depression subscale for Medicaid recipients was $3.9 \pm 1.2$ compared with $3.2 \pm 1.2$ for those who utilized private insurance $(P<0.001)$. Medicaid recipients rated the anxiety subscale highest, with a mean of $3.6 \pm 1.1$ compared with a mean of $3.1 \pm 1.3$ for Medicare beneficiaries $(P=0.006)$. Respondents without insurance had the highest need for assistance with socioeconomic coping, with a mean of $3.6 \pm 1.2$ as compared with a mean of $2.5 \pm 1.3$ for Medicare recipients, $3.2 \pm 1.3$ for Medicaid recipients, and $2.6 \pm 1.2$ for those receiving private insurance $(P=0.0001)$. The scales had the overall means of $3.5 \pm 1.3$ for depression, $3.3 \pm 1.2$ for anxiety, and $2.9 \pm 1.3$ for socioeconomic coping. These means indicate that respondents had the most difficulty coping with depression, followed by anxiety and socioeconomic coping.

The analyses of SLE manifestations revealed that those with muscle pain and hair loss were the most likely to report feelings of SLE-related depression and anxiety. Respondents reporting muscle pain had a mean score of 
$3.8 \pm 1.2$ for depression compared with $2.9 \pm 1.3$ for those who did not $(P<0.001)$. The respondents experiencing muscle pain also had higher levels of anxiety with a mean of $3.7 \pm 1.1$ compared with $2.8 \pm 1.1$ for those who were not experiencing pain $(P<0.001)$. Those who experienced hair loss had a higher level of depression, with a mean of $3.9 \pm 1.2$ compared with $3.1 \pm 1.3$ for those who did not experience this side effect $(P=0.000)$. Respondents reporting chronic symptoms or frequent flares were more likely to have higher psychosocial needs with their depression, anxiety, and socioeconomic coping, as compared with those having infrequent flares. Those who reported frequent flares tested higher for depression, with a mean of $3.8 \pm 1.1(P=0.0000)$ and higher for anxiety, with a mean of $3.7 \pm 1.1(P=0.000)$.

Those who experienced hair loss also had higher levels of anxiety, with a mean of $3.6 \pm 1.2$ compared with $3.0 \pm 1.2$ for those who did not experience this side effect $(P=0.000)$. Similarly, those with muscle pain had higher levels of socioeconomic need, with a mean of $3.0 \pm 1.3$ compared with $2.6 \pm 1.3$ for those who did not $(P=0.006)$.

\section{Multidimensional health locus of control scale findings}

The more respondents perceived they had some control over the illness, the less likely they were to report high levels of depression or anxiety. The mean and median scores for the chance and internal subscales on the Multidimensional Health Locus of Control Scale were: mean $2.84 \pm 1.2$, median 2.7, interquartile range 1.5 , and mean $2.98 \pm 1.2$, median 3.0, interquartile range $=1.6$, respectively, across all patients. Respondents who reported their SLE as having mostly infrequent flares (mean $2.5 \pm 1.2$ ) perceived that they had more control over their health compared with those with chronic symptoms (mean $2.9 \pm 1.2)$ or infrequent flares $(2.8 \pm 0.98$, $P=0.002)$.

In Table 2, the column labeled "Coef" is the slope. This indicates how much the degree of an outcome variable (depression, anxiety, and socioeconomic coping) changes for every point increase in a covariate (eg, chance, internal). The critical locus of control finding was that the more control a patient felt they had over their disease, the less likely they were to report feelings of depression and anxiety, with the specific variances detailed in Table 2 .

\section{Depression}

The most significant locus of control finding was that for those who reported the sensation of having no control over their disease; "chance" (Coef $=0.169, P=0.007$ ) were positively associated with more chronic symptoms (Coef $=0.648, P=0.001)$, frequent flares $($ Coef $=0.796$, $P=0.001)$ and depression $($ Coef $=-0.648, P=0.001)$. A college degree (Coef $=-0.561, P=0.010)$ or advanced degree $($ Coef $=-0.644, P=0.026)$ compared with a high school degree or less was associated with requiring less assistance with depression, as was the case with having Medicare as compared with having private insurance, Medicaid patients required more assistance than those with insurance.

Table 2 Ordinary least squares regression for three outcome variables

\begin{tabular}{|c|c|c|c|c|c|c|c|c|c|}
\hline \multirow{3}{*}{$\begin{array}{l}\text { Dependent } \\
\text { variable } \\
\text { Independent } \\
\text { variable }\end{array}$} & \multicolumn{3}{|c|}{ Depression } & \multicolumn{3}{|l|}{ Anxiety } & \multicolumn{3}{|c|}{ Socioeconomic coping } \\
\hline & \multicolumn{3}{|c|}{$R^{2}=0.29, f=6.4, P=0.00$} & \multicolumn{3}{|c|}{$R^{2}=0.26, f=5.7, P=0.00$} & \multicolumn{3}{|c|}{$R^{2}=0.15, f=2.8, P=0.00$} \\
\hline & Coef. & $\mathbf{t}$ & $\begin{array}{l}\text { 2-tail } \\
\text { significance }\end{array}$ & Coef. & $\mathbf{t}$ & $\begin{array}{l}\text { 2-tail } \\
\text { significance }\end{array}$ & Coef. & $\mathbf{t}$ & $\begin{array}{l}\text { 2-tail } \\
\text { significance }\end{array}$ \\
\hline Internal & -0.071 & -10.16 & 0.247 & -0.019 & -00.32 & 0.749 & 0.005 & 00.07 & 0.941 \\
\hline Chance & 0.111 & 10.87 & 0.063 & 0.138 & 20.36 & 0.019 & 0.051 & 0.73 & 0.466 \\
\hline Hispanic & -0.089 & -0.40 & 0.687 & 0.036 & 0.17 & 0.867 & 0.490 & 10.86 & 0.064 \\
\hline African-American & -0.043 & -0.22 & 0.828 & 0.182 & 0.92 & 0.356 & 0.488 & 20.06 & 0.041 \\
\hline Asian & -0.198 & -0.61 & 0.540 & -0.191 & -0.60 & 0.549 & 0.643 & 10.67 & 0.095 \\
\hline Chronic symptoms & 0.483 & 20.65 & 0.008 & 0.334 & 10.87 & 0.063 & 0.264 & 10.22 & 0.223 \\
\hline Frequent flares & 0.665 & 20.90 & 0.004 & 0.686 & 30.04 & 0.003 & 0.544 & 20.00 & 0.047 \\
\hline Some college & -0.276 & -10.47 & 0.144 & -0.101 & -0.55 & 0.585 & 0.3422 & 10.53 & 0.127 \\
\hline College graduate & -0.440 & -20.14 & 0.034 & -0.404 & -20.00 & 0.047 & 0.101 & 00.41 & 0.680 \\
\hline Advanced degree & -0.543 & -10.93 & 0.054 & -0.157 & -0.57 & 0.568 & 0.204 & 00.61 & $0.54 I$ \\
\hline Medicaid & 0.048 & 00.27 & 0.790 & -0.075 & -00.42 & 0.675 & 0.167 & 0.79 & 0.431 \\
\hline Medicare & -0.589 & -20.86 & 0.005 & -0.4831 & -20.39 & 0.017 & -0.293 & -10.19 & 0.235 \\
\hline No insurance & -0.088 & 00.33 & 0.745 & 0.377 & 10.42 & 0.156 & 10.09 & 30.42 & 0.001 \\
\hline Muscle pain & 0.507 & 30.30 & 0.001 & 0.671 & 40.4 & 0.000 & 0.381 & 20.09 & 0.038 \\
\hline Hair loss & 0.555 & 30.81 & 0.000 & 0.341 & 20.4 & 0.018 & 0.099 & 0.58 & 0.565 \\
\hline
\end{tabular}


The results of the postestimation test indicated that, compared with Medicaid recipients, respondents receiving Medicare had a decreased need for psychosocial assistance with depression $(-0.589, P=0.008)$.

\section{Anxiety}

The following covariates significantly increased the need for psychosocial assistance for feelings of anxiety: chance (Coef $=0.138, P=0.019)$ and frequent flares (Coef $=0.686$, $P=0.003)$ as compared with infrequent flares, and the following covariates decreased the degree of need for psychosocial assistance for feelings of anxiety: college degree (Coef $=-0.538, P=0.011$ ) compared with high school. The results of the postestimation test indicated that compared with respondents with no health insurance, respondents receiving Medicare had a decrease in need for psychosocial assistance for feelings of anxiety (Coef $=0.483, P=0.039)$.

\section{Socioeconomic coping}

Being African-American (Coef $=0.488, P=0.041)$, having muscle pain $($ Coef $=0.380, P=0.038)$ and having no insurance $($ Coef $=1.09, P=0.001)$ all significantly increased the need for psychosocial assistance for socioeconomic needs. The results of postestimation indicated that, compared with respondents with no health insurance, respondents receiving Medicare had a statistically significant decrease in the need for assistance with economic coping $(P=0.034)$.

\section{Medication result}

In analyzing the medication regimens and side effects, it is important to note that respondents could and often did report use of various combinations of drugs. Because the side effects can be a result from any one medication, a combination of SLE medications, or indeed a disease manifestation, the significance of their responses is biased. Because most patients were taking more than one medication at a time, it is difficult to ascertain which specific medications gave rise to which side effects Nevertheless, their perceptions of medication side effects is significant because they may have misinterpreted side effects incorrectly, and may have titrated their own medication regimens based on erroneous perceptions and beliefs. It is also important to glean which side effects are experienced as being the most distressing to these patients, so that treating physicians can assess these issues as they develop or adjust treatment plans. At least one-third of the respondents used hydroxychloroquine, azathioprine, vitamins, methotrexate, steroids, or anti-inflammatory medications. There are many expected and some unexpected side effects of SLE medications that present a myriad of physical and emotional challenges. Most respondents experienced either hair loss $(51.1 \%)$ or weight gain $(32.7 \%)$ as side effects from the use of their respective medications. Table 3 displays the relationship between medications for SLE and the types of side effects respondents experienced from them. Those utilizing hydroxychloroquine and steroids experienced the most side effects. Over two-thirds of those who experienced hair loss $(66.4 \%)$ or weight gain $(67.4 \%)$ were taking hydroxychloroquine $(P=0.02)$.

Another set of interesting findings involved respondents' perceptions of the advantages of SLE medications, as well as their respective emotional preferences for medication treatment plans. The chief reported benefit of these

Table 3 Type of drug by side effects

\begin{tabular}{|c|c|c|c|c|c|c|c|c|c|}
\hline & & \multicolumn{8}{|c|}{ If you had side effects which is most troubling } \\
\hline & & \multicolumn{2}{|c|}{ Hair loss } & \multicolumn{2}{|c|}{ Weight gain } & \multicolumn{2}{|c|}{ Mood swings } & \multicolumn{2}{|c|}{ Nausea } \\
\hline & & $\mathbf{n}$ & $\%$ & $\mathbf{n}$ & $\%$ & $\mathbf{n}$ & $\%$ & $\mathbf{n}$ & $\%$ \\
\hline \multirow[t]{2}{*}{ Hydroxychloroquine } & Yes & 97 & $66.4 \%$ & 62 & $67.4 \%$ & 13 & $39.4 \%$ & 4 & $44.4 \%$ \\
\hline & No & 49 & $33.6 \%$ & 30 & $32.6 \%$ & 20 & $60.6 \%$ & 5 & $55.6 \%$ \\
\hline \multirow[t]{2}{*}{ NSAID } & Yes & 63 & $21.4 \%$ & 47 & $25.5 \%$ & 12 & $17.6 \%$ & 3 & $16.7 \%$ \\
\hline & No & 231 & $78.6 \%$ & 137 & $74.5 \%$ & 356 & $82.4 \%$ & 15 & $83.3 \%$ \\
\hline \multirow[t]{2}{*}{ Steroid } & Yes & 73 & $49.7 \%$ & 57 & $62.0 \%$ & 16 & $47.1 \%$ & 3 & $33.3 \%$ \\
\hline & No & 74 & $50.3 \%$ & 35 & $38.0 \%$ & 18 & $52.9 \%$ & 6 & $66.7 \%$ \\
\hline \multirow[t]{2}{*}{ Methotrexate } & Yes & 19 & $12.9 \%$ & 10 & $10.9 \%$ & I & $2.9 \%$ & I & II.I\% \\
\hline & No & 128 & $87.1 \%$ & 82 & $89.1 \%$ & 33 & $97.1 \%$ & 8 & $88.9 \%$ \\
\hline \multirow[t]{2}{*}{ Azathioprine } & Yes & 14 & $9.5 \%$ & 13 & $14.1 \%$ & 2 & $5.9 \%$ & I & $11.1 \%$ \\
\hline & No & 133 & $90.5 \%$ & 79 & $85.9 \%$ & 32 & $94.1 \%$ & 8 & $88.9 \%$ \\
\hline \multirow[t]{2}{*}{ Cyclosporine } & Yes & I & $0.07 \%$ & 3 & $3.3 \%$ & I & $2.9 \%$ & 0 & $0 \%$ \\
\hline & No & 146 & $99.3 \%$ & 89 & $96.7 \%$ & 33 & $97.1 \%$ & 9 & $100.0 \%$ \\
\hline \multirow[t]{2}{*}{ Vitamins } & Yes & 75 & $51.0 \%$ & 51 & $55.4 \%$ & 15 & $44.1 \%$ & 2 & $22.2 \%$ \\
\hline & No & 72 & $49.0 \%$ & $4 I$ & $44.6 \%$ & 19 & $55.9 \%$ & 7 & $77.8 \%$ \\
\hline
\end{tabular}

Abbreviation: NSAID, nonsteroidal anti-inflammatory drug. 
medications was the reduction in frequency and intensity of flares. When respondents were queried about what they would desire from a new medication for SLE, a majority $(55 \%, \mathrm{n}=207)$ desired fewer flares and almost one-third $(32 \%, n=120)$ desired fewer side effects. Although most respondents experienced side effects of hair loss or weight gain from their medications, they still expressed that their primary desire from a new medication was fewer flares. Almost two-thirds (62.4\%) of those who experienced hair loss desired fewer flares $(P=0.01)$ in a new medication, and $44 \%$ who experienced weight gain also desired fewer flares $(P=0.01)$.

\section{Discussion}

Because this was an exploratory study with a broad focus, it has several inherent limitations and biases. Chief among these is the lack of confirmatory American College of Rheumatology diagnoses. Nevertheless, these were patients who reported themselves as being SLE patients, and were being treated for SLE by rheumatologists. The second critical limitation is that the majority of this sample was on various combinations of SLE medications, and therefore it is difficult to discern which side effects were the results of which treatments. This study highlighted several complex psychosocial reactions that are associated with SLE. Some specific correlations between these factors were uncovered in this cohort. This study reveals an association between chronic symptoms and the likelihood of higher reports of depression and anxiety, but not specifically the cause and dynamic effect. This may be a bidirectional association, in which chronic disease activity influences emotional states or, in fact, that feelings of depression and anxiety triggers more disease activity.

The sequelae of SLE that may predict higher vulnerability for a depressive reaction include more reports of depression attributed to changes in appearance (particularly hair loss and weight gain) and limitations due to SLE (particularly due to muscle pain and joint pain) as was found by Moses ${ }^{12}$ in Australia, Shorthall et a ${ }^{24}$ in England, and Dobkin et al. ${ }^{25}$ This finding was also consistent with that of $\mathrm{Ng},{ }^{26}$ who found that self-esteem for women living with SLE was negatively impacted by changes in body appearance, such as weight gain and hair loss.

When responding to the SLENQ, the sample overwhelmingly reported that the highest general cause of depressive and anxious feelings was changes in appearance and limitations in physical abilities due to SLE, primarily muscle and joint pain. The more chronic the symptoms, the more likely that feelings of depression would arise. The more frequent the flares, the more intense the emotional distress. These correlations between symptomatology and emotional distress are consistent with the findings of other psychosocial researchers in this area, and our sample represents a larger and more ethnically diverse sample. ${ }^{27}$

Learning that African-Americans and Hispanics report a higher vulnerability for SLE-related depression and anxiety, as well as poorer socioeconomic coping than their White and Asian counterparts, is an important finding. This should alert the health care team to integrate social supports into treatment for this population and inform those who develop program service delivery for this population. This finding is consistent with those of other studies reporting health disparities among race and class, ie, the larger burden of disease that Hispanics and African-Americans carry, as well as higher mortality and complication rates. ${ }^{28,29}$ From our data, it is unclear what role language or cultural barriers play in the higher rates of depression reported by Hispanics. This can be further clarified in future research. While all SLE patients should be assessed comprehensively, Hispanic and African-American women may require more psychosocial resources and support, and every effort has to be made to provide culturally competent assessment and intervention. ${ }^{30,31}$ It is critical to appreciate the psychological impact of this illness and the negative side effects of various medical regimens and how these may impact mood changes, and as Moses and $\mathrm{Ng}$ found, feelings of low-self-esteem, capacity for self-care, and medication compliance. ${ }^{12,24-26}$ The Hispanic cohort reported the most emotional distress and reported facing the most socioeconomic distress.

The data also found that the more one perceives control over the illness and the more education one has, the less likely one is to report feeling depressed or anxious about SLE challenges. This is an important finding because there have been no other psychosocial studies which were able to confirm this important relationship. A related study demonstrated that SLE patients with lower levels of education tend to be more anxious, as well as less compliant with visiting their physician. ${ }^{27,28}$ Some possible suppositions about this correlation are that the more educated one is, the better one may be able to understand SLE, and the more resources one may have to cope more adaptively. 3,4

Identifying that those affected by muscle pain and hair loss tend to be more vulnerable to feelings of depression and anxiety is significant for treating physicians, as well as for individual and group counselors. One has to consider the dynamic interplay of the disease manifestations and the 
emotional state, because muscle pain and hair loss could influence depression and anxiety. The converse is also possible, ie, that depression and anxiety could result in both muscle pain and hair loss. Individual and group counseling with SLE patients should allow for exploration and expression of feelings about these specific SLE manifestations and the range of potentially troubling side effects from SLE medications. ${ }^{29}$ Clinical initiatives and program development should emphasize patient education and advocacy because this has been found to be a mitigating factor in both depression and anxiety for this population. ${ }^{31}$

The great majority of SLE patients on medication experience a wide range of side effects, the most prominent of which is hair loss and weight gain, and yet when asked what they wanted from a new medication, the answer was "fewer flares". It is difficult to be definitive regarding certain side effects because some patients may interpret an SLE symptom as a side effect, eg, they may report that Plaquenil ${ }^{\circledR}$ is causing hair loss, but it is also possible that increased SLE activity is the culprit. For many, if they are on steroids, they have active disease, which has its own sequelae.

The prevalence of emotional distress for this population would indicate that intake, assessment, and treatment include inquiry into the psychosocial impact of the illness, with a focus on depression, anxiety, and socioeconomic coping. Assessment should pay particular attention to how a patient has been coping with any changes in appearances and limitations due to SLE, as well as the emotional impact of any side effects they may be experiencing. Any adjustments that can be made to medical regimens with negative side effects should be considered wherever possible. The issue of control for an SLE patient may include information, patient education, and advocacy, as well as emphasizing self-actualization for patients in the use of their resources and psychosocial support. ${ }^{12}$ SLE patients manifesting signs of depressive thinking, anxiety, and difficulty in socioeconomic coping, should be referred to social workers who should employ active therapeutic approaches, such as crisis intervention and task-centered counseling, to combat the unpredictable nature of SLE. ${ }^{32}$

Identifying and confirming that feelings of depression and anxiety occur at significant rates for those living with SLE is critical. Those working in health care should be alerted to which SLE populations are at higher risk, ie, Hispanic and African-American women, and which manifestations trigger the greatest likelihood of feeling depressed and anxious, ie, changes in appearance due to SLE, limitations in physical abilities, joint pain, and weight gain.
Depression and anxiety are broad terms that can reflect very different levels of intensity and frequency for each patient. The SLENQ should be accompanied by the Iverson Depression scale in future research in order to gain more precision for health care treatment. ${ }^{33}$ The overriding study question for future research is - how does the depressed state influence disease activity, disease management, and psychosocial experience of the disease? In addition, further studies should examine SLE patients longitudinally to determine how patients cope adaptively and what strengths (internal and external) aid in the resilience of patients coping with this chronic disease over time.

\section{Conclusion}

This study explored self-reported states of depression and anxiety in SLE patients using the SLENQ instrument, as well as the relationship between perceived sense of control and states of depression and anxiety for the SLE patient. Key findings demonstrated that individuals with SLE were more likely to experience depression and anxiety when there were changes in body appearance due to SLE, and to experience limitations in physical abilities due to SLE, primarily due to muscle and joint pain. The higher the perceived sense of control over SLE, the less likely respondents were to report feeling depressed and anxious. African-American and Hispanic SLE patients reported a higher level of unmet psychological needs than did their other ethnic counterparts. Weight gain and hair loss were the most likely medication side effects, and the most likely to trigger SLE-related depression and anxiety.

Whether the health care provider is the treating physician, nurse, social worker, or any other member of a health care team, it is essential to assess this population for how emotional states may impact disease activity, self-care, and medication compliance. The SLENQ proved a reliable, valid, and comprehensive assessment tool for identifying psychosocial needs related to SLE. The health care team treating this population should be alert to the potential psychosocial impact of SLE, such as depression and anxiety. Health care providers should include comprehensive biopsychosocial screening and assessment wherever possible, and make referrals where needed in order to address the emotional sequelae of living with SLE.

\section{Disclosure}

This research received no specific grant from any funding agency in the public, commercial, or not-for profit sectors. The authors report no conflicts of interest in this work. 


\section{References}

1. Rahman A, Isenberg DA. Systemic lupus erythematosus. NEngl JMed. 2008;358(9):929-939.

2. Pons-Estel GJ, Alarcon GS, Scofield L, Reinlib L, Cooper GS. Understanding the epidemiology and progression of systemic lupus erythematosus. Semin Arthritis Rheum. 2010;39(4):257-268.

3. Wallace DJ. The Lupus Book. New York: Oxford University Press; 2000.

4. Roman MJ, Shanker BA, Davis A. Prevalence and correlates of accelerated atherosclerosis in systemic lupus erythematosus. $N$ Engl J Med. 2003;349(25):2399-2406.

5. Danoff-Burg S, Friedberg F. Unmet needs of patients with SLE. Behav Med. 2009;35(1):5-14.

6. Dobkin P, Fortin PR, Joseph L, Esdaile JM, Danoff DS, Clarke AE. Psychosocial contributors to mental and physical health in patients with systemic lupus erythematosus. Arthritis Care Res. 1998;11(1):23-31.

7. Phillip EJ, Lindner H, Lederman L. Relationship of illness perceptions with depression among individuals diagnosed with lupus. Depress Anxiety. 2009;26(6):575-582.

8. Seawell AH, Danoff-Burg S. Psychosocial research on SLE: A literature review. Lupus. 2004;13(12):891-899.

9. Babul JE, Calderon J, Gonzalez M, et al. Common mental disorders and psychological distress in systemic lupus erythematosus are not associated with disease activity. Lupus. 2011;20(1):58-66.

10. Bachen EA, Chesney MA, Criswell LA. Prevalence of mood and anxiety disorders in women with systemic lupus erythematosus. Arthritis Rheum. 2009;61(6):822-829.

11. Kulczycka L, Sysa-Jedrzejowska A, Robak E. Quality of life and satisfaction with life in SLE patients - the importance of clinical manifestations. Clin Rheumatol. 2010;29(9):991-997.

12. Moses N, Wiggers J, Nicholas C, Cockburn J. Prevalence and correlates of perceived unmet needs of people with systemic lupus erythematosus. Patient Educ Counsel. 2005;57(1):30-38.

13. Schattner E, Shahar G, Leman S, Shakra MA. Depression in systemic lupus erythematosus: The key role of illness intrusiveness and concealment of symptoms. Psychiatry. 2010;73(4):329-340.

14. Carr FN, Nicassion PM, Ishimori ML, et al. Depression predicts selfreported disease activity in systemic lupus erythematosus. Lupus. 2011; 20(1):80-84.

15. McElhone K, Abbott J, Teh LS. A review of health related quality of life in systemic lupus erythematosus. Lupus. 2006;15(10):633-643.

16. Seawell A, Danoff-Burg S. Body image and sexuality in women with and without systemic lupus erythematosus. Sex Roles. 2005;53(11-12): $865-876$.
17. Mendelson C. Managing a medically and socially complex life: Women living with lupus. Qual Health Res. 2006;16(7):982-997.

18. Philip EJ, Lindner H, Lederman L. Confidence in medical care linked to depression in lupus sufferers. J Allied Health. 2009;38(2):106-112.

19. Monaghan SM, Sharpe L, Denton F, Levy J, Schreiber L, Sensky T. Relationship between appearance and psychological distress in rheumatic diseases. Arthritis Rheum. 2007;57(2):303-309.

20. Roman MJ, Shanker BA, Davis A. Prevalence and correlates of accelerated atherosclerosis in systemic lupus erythematosus. $N$ Engl J Med, 2003;349(25):2399-2406.

21. Wallston BS, DeVellis RF. Development of the multidimensional health locus of control (MHLC) scales. Health Educ Monogr. 1978;6(2): 160-170.

22. Lumley T, Diehr P, Emerson S, Chen L. The importance of the normality assumption in large public health data sets. Annu Rev Public Health. 2002;23:151-169.

23. Sawilowsky S, Hillman B. Power of the independent samples t test under a prevalent psychometric distribution. J Consult Clin Psychol. 1993;60(2):240-243.

24. Shorthall E, Isenberg D, Newman S. Factors associated with mood and mood disorders in SLE Lupus. Lupus. 1995;(4):272-279.

25. Dobkin PL, DaCosta D, Fortin PR. Living with lupus: A prospective pan-Canadian study. J Rheumatol. 2001;28(11):2442-2448.

26. Ng Petrus-Chan W. Group psychosocial program for enhancing psychological well- being of people with systemic lupus erythematosus. Lupus. 2007;6(3):75-87.

27. Alarcon GS. Lessons from LUMINA: A multiethnic US cohort. Lupus. 2008;17(11):971-976.

28. Demas KL, Costenbader KH. Disparities in lupus care and outcomes. Curr Opin Rheumatol. 2009;21(2):102-109.

29. Walsh SJ, Gilchrist A. Geographical clustering of mortality from systemic lupus erythematosus in the United States: Contributions of poverty, Hispanic ethnicity and solar radiation. Lupus. 2006;15(10): 662-670.

30. Bogo MA, Tsang K, Lee E. Cross-cultural clinical social work practice. In: Berrold J, editor. Theory and Practice in Clinical Social Work. Los Angeles, CA: Sage Publications; 2011.

31. Tonner C, Trupin L, Yazdany J, Criswell L, Katz P, Yelin E. Role of community and individual characteristics in physician visits for persons with systemic lupus erythematosus. Arthritis Care Res (Hoboken). 2010;62(6):888-895.

32. Reid WJ, Hanrahan P. Recent evaluations of social work: Grounds for optimism. Soc Work. 1982;27(7):328-340.

33. Iverson G. Screening for depression in systemic lupus erythematosus with the British Columbia Major Depression Inventory. Psychol Rep. 2002;90(3 Pt 2):1091-1096.
Journal of Multidisciplinary Healthcare

\section{Publish your work in this journal}

The Journal of Multidisciplinary Healthcare is an international, peerreviewed open-access journal that aims to represent and publish research in healthcare areas delivered by practitioners of different disciplines. This includes studies and reviews conducted by multidisciplinary teams as well as research which evaluates the results or conduct of such teams or

\section{Dovepress}

healthcare processes in general. The journal covers a wide range of areas and welcomes submission from practitioners at all levels, from all over the world. The manuscript management system is completely online and includes a very quick and fair peer-review system. Visit http://www.dovepress.com/testimonials.php to read real quotes from published authors. 